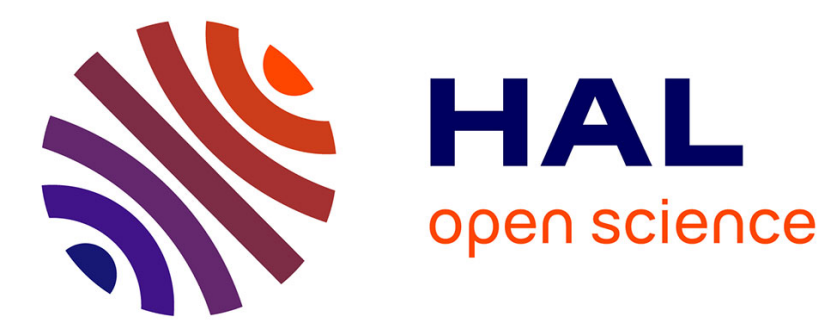

\title{
Revealing load hysteresis based on electronic speckle pattern interferometry and physical mesomechanics
}

\author{
T Sasaki, S Yoshida
}

\section{To cite this version:}

T Sasaki, S Yoshida. Revealing load hysteresis based on electronic speckle pattern interferometry and physical mesomechanics. Physical Mesomechanics, 2012, 15 (1-2), pp.47 - 57. hal-01092767

\section{HAL Id: hal-01092767 \\ https://hal.science/hal-01092767}

Submitted on 5 Mar 2015

HAL is a multi-disciplinary open access archive for the deposit and dissemination of scientific research documents, whether they are published or not. The documents may come from teaching and research institutions in France or abroad, or from public or private research centers.
L'archive ouverte pluridisciplinaire HAL, est destinée au dépôt et à la diffusion de documents scientifiques de niveau recherche, publiés ou non, émanant des établissements d'enseignement et de recherche français ou étrangers, des laboratoires publics ou privés. 


\title{
Revealing load hysteresis based on electronic speckle pattern interferometry and physical mesomechanics
}

\author{
T. Sasaki and S. Yoshida ${ }^{1 *}$ \\ Niigata University, Niigata, 950-2181, Japan \\ ${ }^{1}$ Louisiana University, Hammond, LA, 70402, USA
}

\begin{abstract}
A method to reveal load hysteresis of metal specimens is described. In this method, a low-level tensile- load is applied to a previously loaded specimen, and the resultant displacement field is visualized as a two-dimensional, whole-field image. An optical interferometric technique known as the Electronic Speckle Pattern Interferometry is used for the visualization. The interferometric fringe pattern of the visualized displacement field is analyzed for determination of the degree of deformation from the previous load. A series of experiments have been conducted with aluminum plate specimens for the demonstration of this method. The specimens are preloaded to three stress levels: (A) lower than the yield stress, (B) middle of the plastic regime, and (C) close to the maximum stress. After released from the preload, the specimens are reloaded for fringe pattern analysis. It has been found that the fringe patterns clearly differentiate the three preloading conditions, and that by varying the reload stress level it is possible to reveal the preload stress quantitatively. All experimental observations are explained by deformation dynamics based on physical mesomechanics.

Keywords: plastic deformation and fracture, load hysteresis, electronic speckle pattern interferometry
\end{abstract}

\section{Introduction}

Electronic speckle pattern interferometry (ESPI) is a non-contact and non-invasive method capable of measuring tiny displacement of solid-state objects as a whole-field optical image. With the use of a CCD (charge coupled device) camera and simple software, spatial distributions of the displacement on the objects' surface can be observed as contours of the optical intensity known as the correlation fringes [1]. An important area of application of electronic speckle pattern interferometry in mechanics is non-destructive inspection of objects. Through analysis of the correlation fringes, material behavior such as elastic deformation, plastic deformation and stress concentration can be analyzed on a real-time basis. A number of investigations have been conducted to visualize cracks, the stress field around a crack tip and fatigue damage [2-11]. Furthermore, recent advancement in charge coupled device cameras and image processing technology opens up new exciting applications such as analysis at high-speed deformation [12].

\footnotetext{
* Corresponding author

Prof. Sanichiro Yoshida, e-mail: sanichiro.yoshida@selu.edu
}

In these previous works, the focus is mostly on the analysis of the current status of deformation or prediction of failure. In this study, a different use of electronic speckle pattern interferometry is demonstrated; rather than analyzing the current deformation status or predicting the future behavior of a material, the electronic speckle pattern interferometry technique is used to reveal loading hysteresis. The idea is that by applying a minimum test load to a previously deformed specimen and analyzing the resultant fringe pattern, the level of the loading hysteresis is diagnosed in light of physical mesomechanical interpretation of deformation and fracture [13-15]. Our preliminary studies $[16,17]$ have demonstrated that preloaded aluminum sheet specimens present fringe patterns clearly different from non-preloaded specimens of the same material and dimension, and that depending on the preload stress level, the fringe patterns observed in the reload can be classified into different stages. In addition, the characteristic features of the classified fringe patterns have been qualitatively explained in connection with the level of deformation expected in the respective stages. The aim of this paper is to investigate this idea in a more quantitative fashion, and discuss the physics underlying the classified fringe patterns. 


\section{Experimental}

\subsection{Experimental procedure}

To investigate the above idea of using electronic speckle pattern interferometry to reveal loading hysteresis, the following experiments were conducted. In all the experiments, a tensile load at a constant crosshead speed of $0.02 \mathrm{~mm} / \mathrm{s}$ was applied.

Experiment 1. Single loading till failure

In this experiment, the tensile load was applied to the specimen until it failed. The purpose of this experiment was to acquire baseline data of fringe images which were compared with those from other experiments described below.

Experiment 2. Reload with low level

In this experiment, preloaded specimens were prepared with application of the tensile load up to three different stress levels: $A, B$ and $C$ (Fig. 1). After released from the preload, each preloaded specimen was reloaded up to a stress level of $30 \mathrm{MPa}$, about a half of the yield stress (the stress level at which the material is definitely deforming plastically). The purpose of this experiment was to examine if the preloading level could be revealed from the fringe patterns obtained in the reloading.

Experiment 3. Reload until failure

In this experiment, the preloaded specimens $A-C$ were reloaded until they failed. The purpose of this experiment was to examine if the preloaded specimens show different fringe patterns from Experiment 1 (i.e., non-preloaded specimens) at each stage of deformation toward failure.

\subsection{Specimen}

The material used in this study was pure aluminum (99.99\%). A $0.5 \mathrm{~mm}$-thick aluminum sheet was cut into a tensile specimen of $4 \mathrm{~mm}$ in gauge width and $25 \mathrm{~mm}$ in gauge length. Subsequently, the specimens were annealed at $400{ }^{\circ} \mathrm{C}$ for $30 \mathrm{~min}$. The crystal grain size after annealing was approximately $70 \mu \mathrm{m}$.

\subsection{Loading condition and the stress-strain curve}

Figure 1 shows a typical stress-strain curve obtained in Experiment 1 Single loading till failure. Based on this stressstrain curve, three preloading conditions for Experiment 2 and 3 were determined: $A-30 \mathrm{MPa}, B-55 \mathrm{MPa}$, and $C-65 \mathrm{MPa}$. The solid circles in Fig. 1 depict these three conditions. The residual strains after releasing the three preloads were $0.01,0.1$ and 0.2 , respectively. Being annealed aluminum, the specimen did not show a clear yield point on the stress-strain curve. Based on the level of the abovementioned residual strain and the linearity of the stress-strain curve, it is reasonable to assume that the deformation is practically elastic under preload condition A. On the other hand, under preload conditions $B$ and $C$, it is clear that the specimen is deformed plastically. For the maximum stress

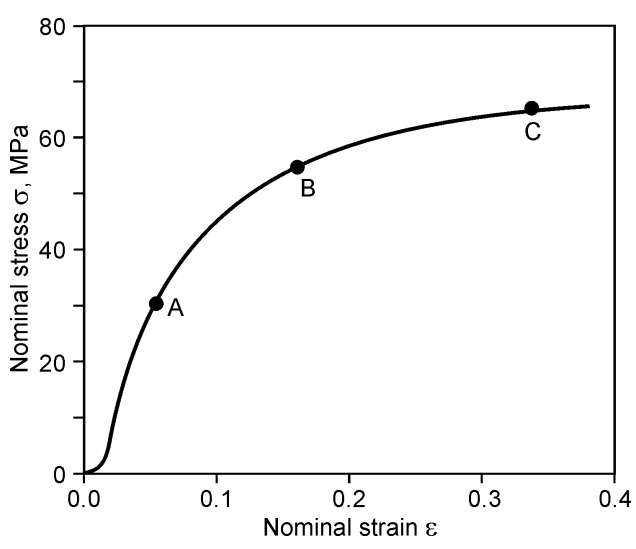

Fig. 1. Preload condition

level of the reload test, $30 \mathrm{MPa}$, the same load as the elastic preload level $A$, was selected. The crosshead speed in the preload and reload was constant at $0.02 \mathrm{~mm} / \mathrm{s}$.

\subsection{Displacement measurement with electronic speckle pattern interferometry}

Figure 2 illustrates the experimental arrangement used in this study. A dual beam electronic speckle pattern interferometry setup [18] sensitive to horizontal $(x)$, in-plane displacement was arranged in front of the aluminum specimen, which was mounted on a tensile machine that applied tensile loads in the positive $x$ direction. The light source was a continuous wave, helium neon laser oscillating at $632.8 \mathrm{~nm}$. The laser beam was split into two paths by a beam splitter. After being expanded by beam expanders, the two beams illuminated the specimen at the same angle of incidence at $45^{\circ}$. This caused diffused reflection of the laser beams on the specimen surface, each forming a speckle pattern on the image plane of the CCD camera that captured the image of the specimen. Thus each pixel of the CCD camera received optical intensity resulting from coherent superposition of the speckles due to the two laser beams. When the specimen was stretched by the tensile machine, different parts of the specimen surface displaced differently, causing the corresponding speckles on the image plane move accordingly. As points on the specimen surface displaced

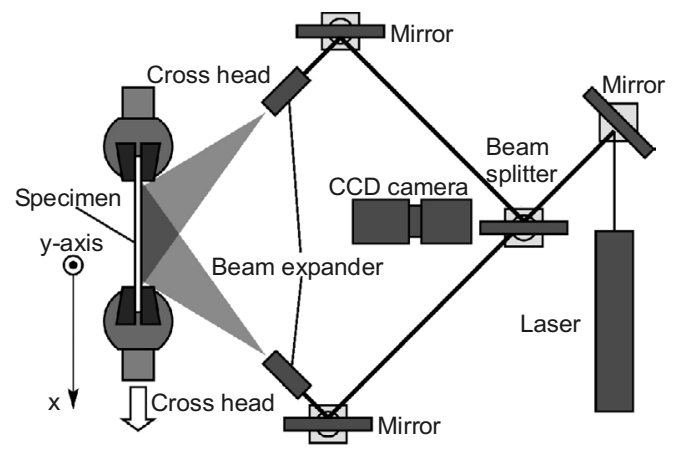

Fig. 2. Experimental arrangement 
in the positive $x$ direction, the optical path length to the image plane increased for the left laser beam (the lower beam in Fig. 2), and decreased for the other laser beam. Consequently, the brightness of the superposed speckle field changed as the specimen experienced deformation. Thus, this setup had sensitivity of the displacement along the $x$ axis.

The image of the specimen during the tensile test was captured by the CCD camera at a constant rate of $15 \mathrm{fps}$ (frames per second). The images were recorded into computer memory, and the change in the brightness of speckles was calculated numerically by subtracting each frame of image from a frame captured at a later frame, typically 3 frames later $(0.2 \mathrm{~s})$. Consequently, the displacement distribution on the surface was observed as contours called the fringes.

In this type of electronic speckle pattern interferometry setup, when each part of the specimen displaces in proportion to the distance from the stationary end of the specimen under elastic deformation, the resultant fringe pattern consists of vertical (perpendicular to the tensile axis) straight lines with equal intervals. On the other hand, when the displacement along the tensile direction ( $x$-axis) solely depends on the coordinate perpendicular to it ( $y$-axis), such as the case of shear deformation or rigid body rotation, the fringe pattern consists of horizontal straight lines [19]. If the deformation is a combination of these, which is normally the case, the resultant fringes are mixture of these patterns.

\section{Experimental results}

\subsection{Experiment 1. Single loading till failure}

Figure 3 shows fringe patterns obtained at several stress levels in Experiment 1. The images in this figure represent deformation resulting from the deformation interval of $0.2 \mathrm{~s}$, i.e., the subtraction of a speckle pattern formed at a certain time step from the speckle pattern formed $0.2 \mathrm{~s}$ later. As the crosshead speed of the tensile machine is $0.02 \mathrm{~mm} / \mathrm{s}$, the total elongation during this deformation interval is $0.02(\mathrm{~mm} / \mathrm{s}) \cdot 0.2(\mathrm{~s})=0.004 \mathrm{~mm}$. Consequently, the displacement corresponding to a pair of neighboring fringes (called the unit fringe displacement) is $632.8(\mathrm{~nm}) \cdot \sin 45^{\circ} \cdot 2=$ $=447.5 \mathrm{~nm}$. Here, $632.8 \mathrm{~nm}$ is the laser wavelength and $45^{\circ}$ is the angle of incidence of the two laser beams on the specimen surface. Thus the total number of fringes corresponding to this load interval is $0.004(\mathrm{~mm}) / 447.5(\mathrm{~nm})=$ $=9$ fringes (called the reference fringe number).

When the stress is low (Fig. 3(a), $\sigma<5 \mathrm{MPa}$ ), the fringes are unclear and some of them are slightly tilted from the vertical position. The number of fringes observed over the horizontal span of the specimen, six fringes, is less than the above defined reference fringe number. It is suspected that this is because part of the deformation occurs out of the active interference zone; it is likely that at this initial stage of loading, the deformation is substantial near the grips of the tensile machine where the sides of specimen are curved (from the parallel part to the wider part). In these regions, the laser beam is partially blocked by the nearby grip. Consequently, the interference does not occur and fringes are not formed. The active interference zone is in between the two vertical straight lines observed near the two ends of the specimen in all images of Fig. 3.

At the stress level of $30 \mathrm{MPa}$ (Fig. 3(b)), the observed total number of fringes is approximately equals the reference fringe number, indicating that the deformation occurs in the active interference zone. This stress level of $30 \mathrm{MPa}$ is the same as the preload condition $A$. The stress-strain curve shown in Fig. 1 indicates that at this stress level the deformation is still in the range traditionally known as the elastic regime. From this viewpoint, it is not surprising that the deformation is stably continues in the parallel part of the specimen. However, the strain distribution observed from the fringe pattern shows some behavior that is different from the traditional concept of elastic deformation. In Fig. 3(b), the fringes are basically vertical $\left(\partial \xi_{x} / \partial y \cong 0\right.$, where $\xi_{x}$
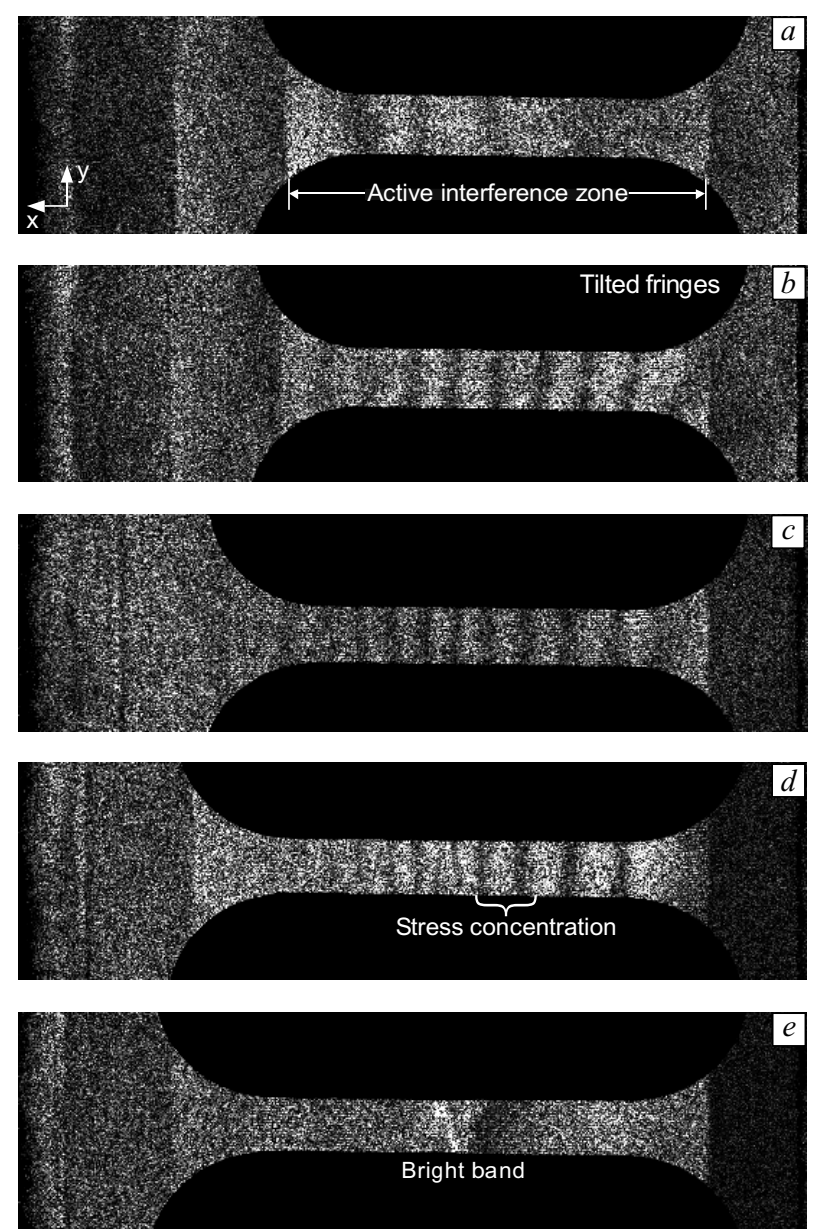

Fig. 3. Fringe patterns in the annealed aluminum (Experiment 1). The deformation interval is $0.2 \mathrm{~s}$; $\sigma<5 \mathrm{MPa}(a), \sigma=30(b), 55(c), 65 \mathrm{MPa}$ $(d)$, before failure $(e)$ 
denotes the horizontal displacement) toward the left end of the specimen and somewhat tilted $\left(\partial \xi_{x} / \partial y>0\right)$ near the right end. (Consider the rightmost fringe oriented in the direction of 1 o'clock. Say the horizontal displacement $\xi_{x}$ of this fringe is one unit fringe displacement $\xi_{x 0}$. Since the right grip is stationary, the next fringe on the left is two unit displacements $\left(2 \xi_{x 0}\right)$. It is thus observed that in the region between these two fringes, the displacement is higher (more leftward) as going up (increasing $y$ ) from the bottom of the specimen. Call this additional displacement toward the top of the specimen the excess stretch.) As rotation around the $z$-axis is given by $\omega_{z}=\partial \xi_{y} / \partial x-\partial \xi_{x} / \partial y$, positive $\partial \xi_{x} / \partial y$ indicates $\omega_{z}<0$; thus the right end part of the specimen rotates counter-clockwise. (The sign of $\omega_{z}$ also depends on $\partial \xi_{y} / \partial x$, but it is reasonable to assume that being perpendicular to the tensile axis, $\xi_{y}$ is considered to be much smaller than $\xi_{x}$, and thereby $\partial \xi_{x} / \partial y$ is the dominant term of $\omega_{z}$.) Alternatively, it can be interpreted that the top edge of the specimen displaces more than the bottom toward the right end of the specimen, or the top side is weaker than the bottom.

It is interesting to note that the specimen experiences rotations at the stage where the stress-strain curve is still nearly linear. It indicates that although the relationship between the averaged stress and strain observed as the stressstrain curve appears to be linear, locally the deformation can be non-linear $\left(\partial \xi_{x} / \partial y\right.$ varies as a function of $\left.x\right)$. It is known that plastic deformation typically occurs as crystal grains slip in a certain direction determined by the crystal orientation and that therefore the deformation is accompanied by rotation and shear deformation of each crystal grain. From this viewpoint, it is fair to say that at the stage where the stress-strain curve appears to be linear, plastic deformation is already initiated. This agrees with basic postulate of physical mesomechanics [13].

When the stress level is increased to $55 \mathrm{MPa}($ Fig. 3(c)), the fringe pattern shows two distinctive differences from Fig. 3(b). First, the fringes are more vertical, indicating that the specimen is less rotational. Second, the total number of fringes exceeds the reference fringe number indicating that some part of the specimen experiences contraction. As discussed in more detail in a later section, this can be interpreted as follows: the excess stretch initiated at the top side of the specimen propagates downward across the width of the specimen, and at the stage of Fig. 3(c), it completes the propagation. In other words, the weakening effect bridges the width of the specimen. It is likely that this bridging phenomenon occurs rather instantaneously at the moment when the excess stretch reaches the bottom end of the specimen. Consequently, the two sides of the specimen divided by the bridged portion recoil in mutually opposite directions. When this happens, the left end of the right portion and the right end of the left portion of the specimen experience contraction. (Imagine that you cut a horizontally stretched spring.
The two portions of the resultant cut-off springs will contract.)

In the next stage represented by Fig. 3(d), the fringes are basically vertical, similarly to Fig. 3(c). However, the pattern is clearly different from Fig. 3(c) in that the fringes are much less evenly distributed horizontally. In particular, the fringes are less distinctive in the left half of the specimen, and somewhat concentrated near the boundary of the left half and the right half of the specimen where fringes are more distinctive. This clearly indicates that the strain is being concentrated in this central zone.

The tendency of strain concentration is further enhanced towards the failure of the specimen. As shown in Fig. 3(e), eventually all the fringes are concentrated in a small region and appear as a bright pattern. It is interesting to note that the location of such a bright pattern can vary at the late stage of deformation, but eventually it becomes stationary and the specimen always fails along the bright pattern. This phenomenon has been observed in various materials [20]. From these observations, it can be said that plastic deformation is characterized as a process where the strain gets concentrated. The negative elongation can be understood as strain concentration occurring at a certain location of the specimen causes stress relaxation, making that part of the material recoil. In addition, failure can be characterized as the final stage of strain concentration.

The observation in the fringe patterns associated with the development of plastic deformation can be argued in a quantitative fashion as well. Figure 4 plots the fringe interval as a function of the strain; here the solid circle represents the mean value and the error bar the standard deviation of the fringe interval. The mean fringe interval decreases up to a strain of about 0.1 , above which it is nearly constant at between 1.5 and $2 \mathrm{~mm}$. On the other hand, the standard deviation clearly increases beyond the strain of 0.1 . Figure 1 indicates that the strain value of 0.1 is where the stressstrain curve considerably deviates from the initial linear part; hence it is reasonable to say that beyond this strain value

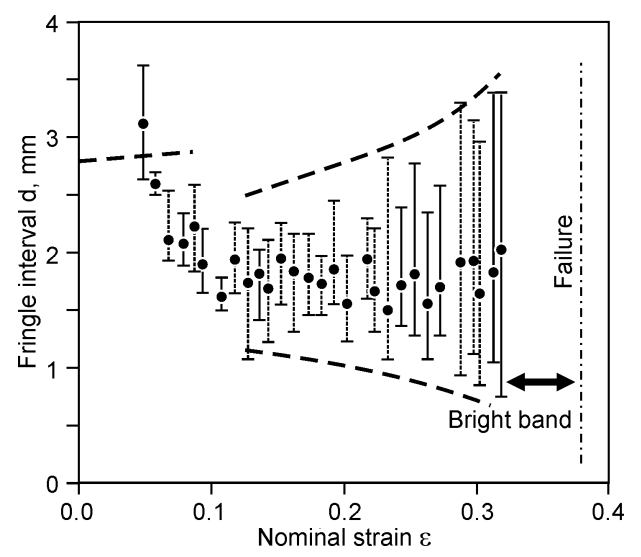

Fig. 4. Relationship between fringe interval and nominal strain in the specimen as annealed 
the specimen is dominantly being plastically deformed. Such behavior of the mean value and standard deviation of the fringe interval support the above arguments of plastic deformation made in connection with the fringe patterns as follows. Since the gauge length of the specimen is $25 \mathrm{~mm}$, the fringe interval corresponding to the reference fringe number of 9 is around $2.5 \mathrm{~mm}$. Figure 4 indicates that at the strain level 0.1 and higher, the mean fringe interval is in between 1.5 and $2 \mathrm{~mm}$, which is considerably lower than $2.5 \mathrm{~mm}$. This means that beyond this point, the total number of the fringes exceeds the reference fringe number and that therefore part of the specimen experiences contraction. The increase in the standard deviation observed at the strain level 0.1 and higher indicates the strain concentration; as the plastic deformation develops the fringes becomes denser at a certain part than the rest of the specimen.

\subsection{Experiment 2. Reload with low level}

Figure 5 shows the stress-strain curves of Experiment 2 for the three different preload conditions. Here the dashed line is the stress-strain curve of Experiment 1 for comparison. It is observed that the stress-strain curves in Experiment 2 are as linear as Experiment 1. Moreover, the stressstrain curves in Experiment 2 have a somewhat greater slope than Experiment 1, and the linear range is longer as well. These observations can be interpreted as that the preload removes the nonlinearity of the specimen so that when it is reloaded the stress-strain curves are actually more linear than the single loading case.

While the stress-strain curves are similar, the fringe patterns observed in Experiments 1 and 2 are substantially different from each other, indicating the possibility of finding the load hysteresis via examination of the fringe pattern observed under low-level reload. Figure 6 shows fringe patterns observed in Experiment 2 for the three different pre-

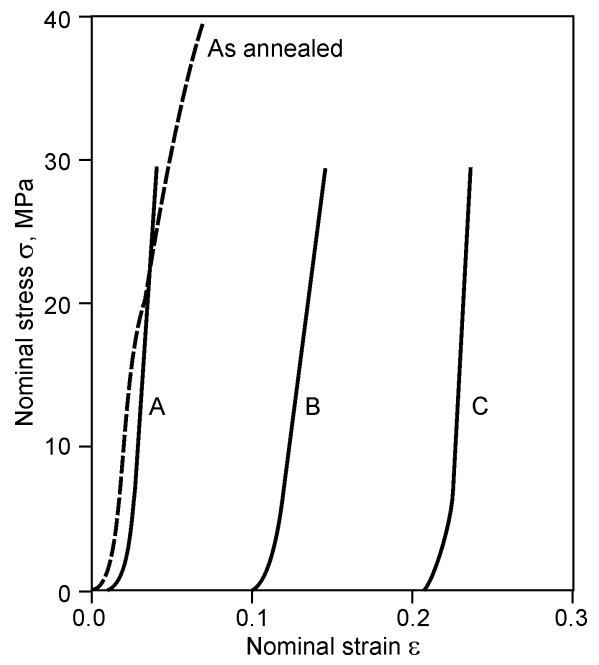

Fig. 5. Stress-strain curve in reload test, $\sigma_{\mathrm{p}}=30(A), 55(B), 65 \mathrm{MPa}(C)$
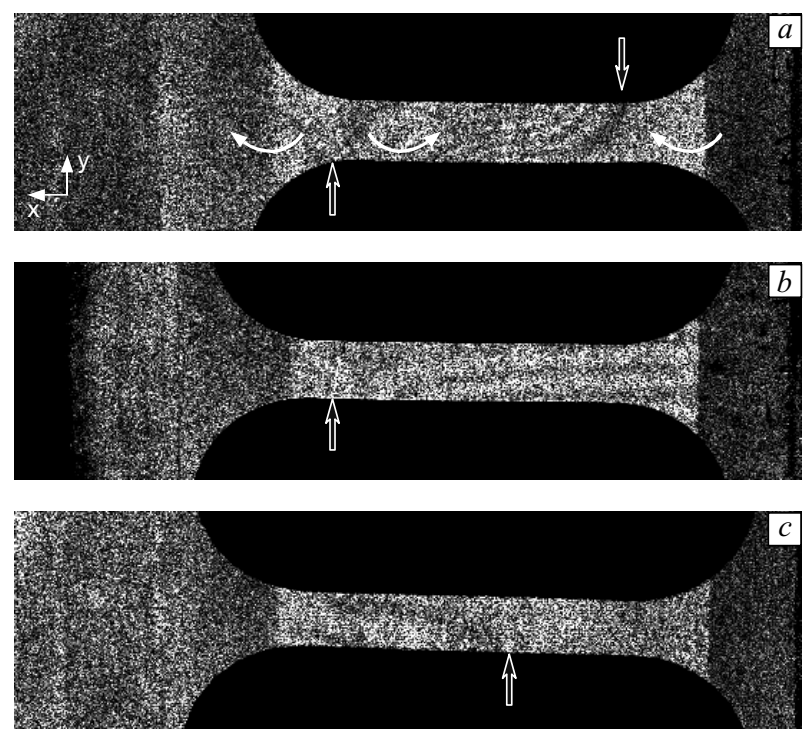

Fig. 6. Fringe patterns obtained in Experiment 2 at the reload stress level of $30 \mathrm{MPa}$. Preload condition $A, 30 \mathrm{MPa}(a) ; B, 55 \mathrm{MPa}(b)$; $C, 65 \mathrm{MPa}(c)$

load conditions. The reload stress level at which these fringe patterns were obtained is $30 \mathrm{MPa}$, or the same stress level at which fringe pattern Fig. 3(b) was obtained in Experiment 1. Notice that while the fringes in Fig. 3(b) are all essentially vertical and approximately parallel to one another, fringes shown in Fig. 6 are basically horizontal and equidistant-parallel under all preload conditions. Moreover, as the preload stress level increases as going from condition $A(30 \mathrm{MPa})$ to $C(65 \mathrm{MPa})$, the fringes are more horizontal except for some points where the fringes look spouting out from the lower/upper side of the specimen to the other side (these locations are indicated with thick arrows in Fig. 6). Generally speaking, an equidistant-horizontal fringe system observed in a horizontally sensitive electronic speckle pattern interferometry setup indicates that the object experiences bodily rotation $[19,21]$. Thus, it is interpreted that the specimen rotates in opposite directions on the two segments bounded by the points where the fringes are spouting out (called the segmental bodily rotation). The circular arrows in Fig. 6(a) indicate the directions of the segmental bodily rotations.

\subsection{Experiment 3. Reload until failure}

Figure 7 shows the stress-strain curve observed in Experiment 3 with a specimen preloaded at $55 \mathrm{MPa}$ (preload condition $B$ ). The labels $a-c$ denote three representative reload stress levels at which the three fringes in Fig. 8 are formed for analysis. Note that stress levels $a$ (25 MPa), $b$ $(55 \mathrm{MPa})$ and $c(65 \mathrm{MPa})$ are, respectively, lower, the same, and higher than the preload stress level of $55 \mathrm{MPa}$. The following observations can be drawn: when the reload stress level is lower than the preload stress level (Fig. 8(a)), the fringe pattern is similar to Fig. $6(b)$, the fringe formed at 


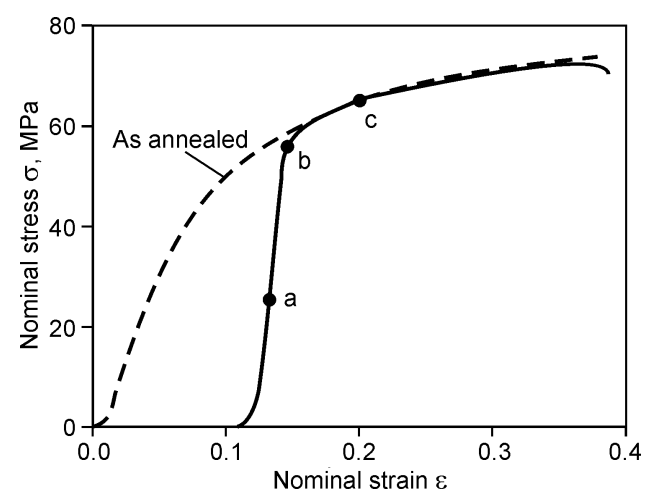

Fig. 7. Stress-strain curve in deformation until failure after preloading

the reload stress of $30 \mathrm{MPa}$ with the same preload stress (i.e., preload condition $B$ ). On the other hand, when the reload stress level exceeds the preload level (Fig. 8(c)), the fringe pattern appears to be similar to Fig. $3(d)$, the fringe formed at the same stress level for the unpreloaded specimen. When the reload stress level is at the same as the preload (Fig. 8(b)), the fringe pattern is transitional from Fig. 8(a) to Fig. 8(c).

\section{Discussions}

\subsection{Summary of observations}

The above arguments on the development of deformation based on the observations under Experiment 1-3 can be summarized as follows. From the very early stage of deformation where the stress-strain curve is linear, some part of the specimen stretched more than the rest of the specimen. Fringe patterns observed in Experiment 1 indicates that such an excess stretch is initiated at one of the horizontal sides of the parallel part of the specimen, and
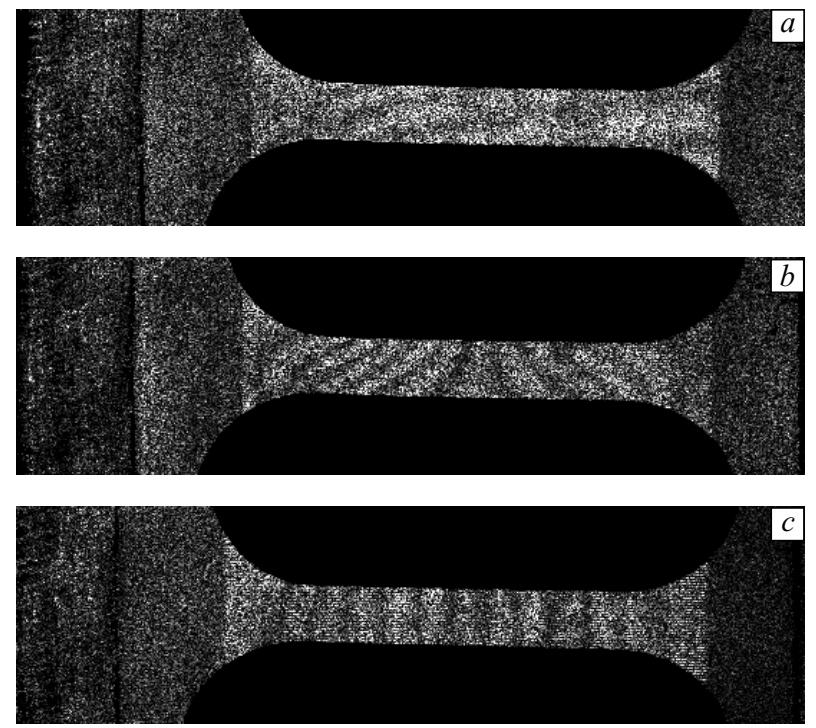

Fig. 8. Fringe pattern in reloading preloaded specimen at $55 \mathrm{MPa}$ till failure. Reload stress $25(a), 55(b), 65 \mathrm{MPa}(c)$ propagates across the width of the specimen. This excess stretch on one side results in the segmental bodily rotations of the specimen. The cause of the excess stretch cannot be inferred from the fringe patterns, but from the observation that it is accompanied by the reduction of stress increase in the stress-strain curve, it is natural to hypothesize that the excess stretch is associated with irreversible atomic rearrangement comprehensively termed dislocations. Consequently, the resultant local strain is permanent. Further, as the elasticity is represented by force proportional to displacement, generally termed the spring force, it is natural to term this irreversible process the breakage of spring, meaning that the spring representing the elastic force of the region where the permanent strain occurs loses elasticity.

Fig. 9 illustrates this process schematically, where the excess, permanent stretch is denoted by ' $X$ ' indicating a broken spring. In this illustration, the spring breakage is initiated at the top atomic row in the left half of the specimen, and propagates downward, causing the right side of the breaking point of the left half to rotate clockwise. Being gripped by the tensile machine, the two ends of the entire specimen is constrained not to be able to displace vertically. Consequently, the clockwise rotation of the right side of the left half of the specimen induces counterclockwise rotation on the right half of the specimen, stretching more the bottom end of the specimen as indicated by Fig. 9. The two arrows in Fig. 6(a) denoting the boundary of the central segmental bodily rotation of the specimen correspond to the two conjugate spring breakages in Fig. 9(a). Once the spring breakage completes its propagation across the width of the specimen, the specimen gains freedom to displace vertically at the location where the propagation completes. That is why Figs. 6(b) and (c) show only one boundary of opposite rotations.
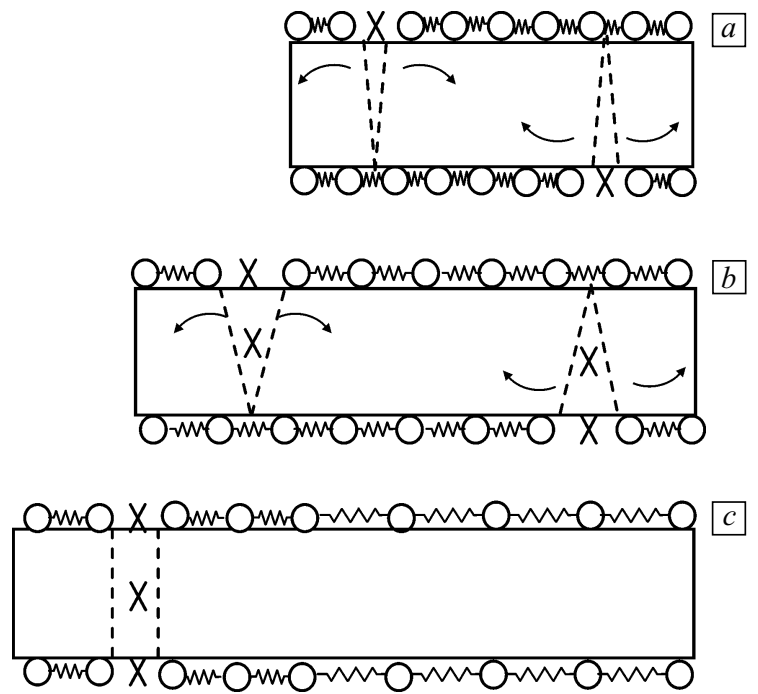

Fig. 9. Schematic illustration of generation and propagation of permanent stretch 
When the specimen is released from the load after the spring breakage propagates across the specimen to some extent, only the elastic stretch is relaxed; the permanent stretch remains. Therefore, when the specimen is reloaded at a stress level lower or the same as the stress level at which the specimen is released, only the elastic stretch resumes. Consider a situation where a specimen is released from the load when the permanent strain is greater on the top side of the specimen than the bottom, as in Fig. $9(b)$. For the sake of argument, say that the top side of the specimen can be represented by two stretchable springs and one unstretchable spring (as a consequence of the permanent stretch) whereas the bottom side by three stretchable springs, and that the reload stress causes total strain of $15 \%$ at the right end of the specimen gripped by the dynamics head of the tensile machine. Figure 10 illustrate the situation where $x_{0}, \ldots, x_{3}$ indicates the locations of the ends of the springs. The top illustration $(a)$ represents the situation before the specimen is pulled for the first time, $(b)$ the situation when the specimen is released from the preload, and (c) the situation at a certain point when the specimen is being reloaded. The top middle spring enclosed by a box (in $(b)$ and $(c)$ ) represents the unstretchable spring.

When this specimen is reloaded, since all the three springs at the bottom are stretchable, the total elongation is shared by the three springs; consequently, each spring stretches by $15 \% / 3=5 \%$ of the length of the specimen. On the other hand, along the top of the specimen, the same total elongation is shared by the two stretchable springs, i.e. the stretch is $7.5 \%$ per spring. Consequently, the positions of the end of the springs along the top and bottom side become different as shown in Table 1. Because of the unstretchable spring, the distance between $x_{1}$ and $x_{2}$ along the bottom of the specimen is greater than the top; equivalently speaking, the left segment of the specimen between $x_{0}$ and $x_{1}$ rotates clockwise, and the other segment between $x_{2}$ and $x_{3}$ counter clockwise. The dashed line in

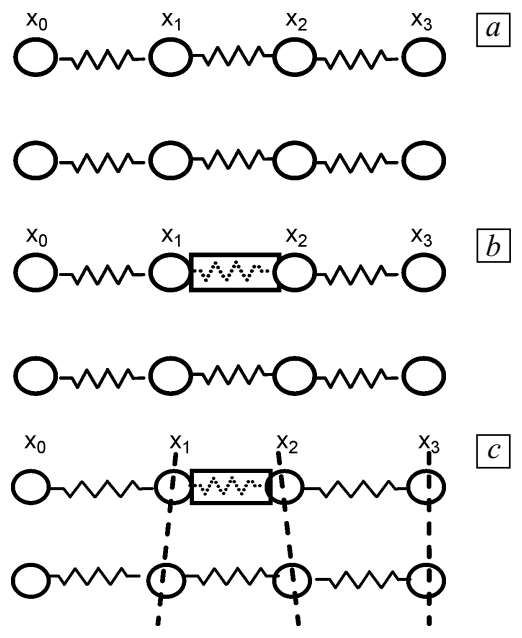

Fig. 10. Broken spring in reloading: preload $(a)$, release from preload (b), reload (c)
Positions of spring ends at top and bottom rows

\begin{tabular}{c|c|c|c|c}
\hline & $x_{0}$ & $x_{1}$ & $x_{2}$ & $x_{3}$ \\
\hline Top & 0 & $1.075 l_{0}$ & $2.075 l_{0}$ & $3.150 l_{0}$ \\
\hline Bottom & 0 & $1.050 l_{0}$ & $2.100 l_{0}$ & $3.150 l_{0}$ \\
\hline
\end{tabular}

Fig. 10 indicates the situation. This is the cause of the fringe patterns indicating non-zero $\partial \xi_{x} / \partial y$ (called the differential horizontal displacement).

This segmental bodily rotation due to the differential horizontal displacement occurs in different fashions among the three experiments discussed above. In Experiment 1 where the annealed specimen is pulled for the first time, the fringes are formed as irreversible atomic rearrangement (the formation of unstretchable springs) occurs. Consequently, the resultant segmental bodily rotation is observed in the fringe patters as a step-by-step process (as opposed to a jump from Fig. 10(b) to Fig. 10(c) in the case of reloading). The vertical fringes continue to tilt as the nearby specimen experiences the irreversible atomic rearrangement. After the irreversible atomic arrangement completes propagating across the width of the specimen as discussed under Section 3.1 (where the irreversible atomic arrangement is referred to as "the excess stretch"), the fringe patterns stop showing the segmental bodily rotation; fringes become more vertical.

In Experiment 2, the irreversible deformation is observed in a different fashion from Experiment 1. The fringe pattern presents the segmental bodily rotation resulting from the differential horizontal displacement accumulated during the preloading, as opposed to the step-by-step rotations in the case of Experiment 1. When the preload stress is low, low-level irreversible atomic rearrangements occur at different locations of the specimen. Therefore, local, smallscale segmental bodily rotations occur at multiple locations, resulting in a wavy fringe pattern like Fig. 6(a). When the preload stress is high, the irreversible atomic rearrangement bridges before the specimen is released from the preload. As the bridging effect occurs, the two segments of the specimen divided by the bridged location are relaxed from the stress. Consequently, when reloaded, the fringe pattern shows mutually opposite bodily rotations on the two segments bounded by the bridging location. Fringe patters Fig. $6(b, c)$ represent such situations. Note that the horizontal fringes are straighter than Fig. 6(a), because inside the two segments the material is barely deformed (more rigid-body like).

In Experiment 3, the situation is the same as Experiment 2 until the reload stress exceeds the preload stress (Fig. 8(a)). When the reload stress becomes greater than the preload stress, the formation of new irreversible atomic rearrangement resumes. Consequently, the fringe patterns (Fig. 8(c)) become similar to Experiment 1 where new irreversible atomic rearrangement occurs continuously. 


\subsection{Physical mesomechanics}

The above-discussed experimental observations are well explained from the viewpoint of physical mesomechanics. In this section, the gist of the theory is quickly reviewed and the above-discussed observation is interpreted based on the physical mesomechanical dynamics.

Physical mesomechanics postulates that in the plastic regime deformation is globally nonlinear but locally elastic. Based on this postulate and the concept known as local (gauge) symmetry [22], it introduces an additional vector field to the displacement field. This additional field can be characterized by two field variables; $\mathbf{v}$ representing the translational displacement of the local volume element at a global coordinate point, and $\omega$ representing the rotational displacement of the element at the same coordinate point. From the field theoretical standpoint, $\mathbf{v}$ and $\boldsymbol{\omega}$ can be viewed as four-dimensional gradient of the potential associated with the gauge that makes the linear elastic theory symmetric under the condition of the global non-linearity. More details regarding the field theoretical aspects of physical mesomechanics can be found in $[14,15,23]$.

With these field variables, the dynamics of plastic deformation can be concisely expressed by the following set of equations:

$$
\begin{aligned}
& \nabla \cdot \mathbf{v}=j^{0}, \\
& \nabla \times \boldsymbol{\omega}=-\varepsilon \mu \frac{\partial \mathbf{v}}{\partial t}-\mathbf{j} .
\end{aligned}
$$

Here, $\varepsilon$ and $1 / \mu$ are the density and shear modulus of the material, and $j^{0}$ and $\mathbf{j}$ are the time and space component of the symmetry charge associated with the gauge, $\mathbf{v}$ and $\boldsymbol{\omega}$ are related to each other by the following equation:

$$
\frac{\partial \boldsymbol{\omega}}{\partial t}=\nabla \times \mathbf{v}
$$

Defining a quantity

$$
\rho=\varepsilon j^{0}
$$

Equation (2) can be rewritten as follows:

$$
\varepsilon \frac{\partial \mathbf{v}}{\partial t}=-\frac{1}{\mu}(\nabla \times \boldsymbol{\omega})-\frac{\mathbf{j}}{\mu} .
$$

Derivation of Eqs. (1)-(5) and the physical meanings of these equations are described elsewhere [15, 23].

Equation (5) can be viewed as an equation of motion governing the dynamics of unit volume [24]. The left-hand side represents the change in momentum of the unit volume over time. The first term on the right-hand side represents the shear force, which is restoring force and responsible for the energy conservative, wave dynamics $[15,25]$ in the plastic regime. In a two-dimensional model like this study, the shear force can be explicitly expressed as follows:

$$
\begin{aligned}
F_{x} & =-\frac{1}{\mu}(\nabla \times \boldsymbol{\omega})_{x}=-\frac{1}{\mu} \frac{\partial \omega_{z}}{\partial y}= \\
& =\frac{1}{\mu} \frac{\partial}{\partial y}\left(-\frac{\partial \xi_{y}}{\partial x}+\frac{\partial \xi_{x}}{\partial y}\right),
\end{aligned}
$$

$$
\begin{aligned}
F_{y} & =-\frac{1}{\mu}(\nabla \times \omega)_{y}=-\frac{1}{\mu}\left(-\frac{\partial \omega_{z}}{\partial x}\right)= \\
& =\frac{1}{\mu} \frac{\partial}{\partial x}\left(\frac{\partial \xi_{y}}{\partial x}-\frac{\partial \xi_{x}}{\partial y}\right) .
\end{aligned}
$$

If viewed in fringe patterns formed by a horizontally sensitive interferometer, since the fringes represent only the horizontal component of displacement $\xi_{x}$, the above two force components become

$$
\begin{aligned}
& F_{x}=\frac{1}{\mu} \frac{\partial}{\partial y} \frac{\partial \xi_{x}}{\partial y}, \\
& F_{y}=-\frac{1}{\mu} \frac{\partial}{\partial x} \frac{\partial \xi_{x}}{\partial y} .
\end{aligned}
$$

Equation (6) indicates that when the horizontal ( $x$ ) component of the shear force in the plastic regime is active, the corresponding fringes are curved $\left(\partial^{2} \xi_{x} / \partial^{2} y \neq 0\right)$; when the vertical component of the shear force is active, the fringes can be linear but not vertical $\left(\partial \xi_{x} / \partial y \neq 0\right)$, and its slope varies as going horizontally $\left(\partial^{2} \xi_{x} / \partial x \partial y \neq 0\right)$.

The second term on the right-hand side of Eq. (5) represents the longitudinal effect acting on the momentum change of the unit volume. In the elastic regime, this term represents the longitudinal elastic force proportional to the displacement from equilibrium position. In the plastic regime, it represents energy dissipation associated with the permanent stretch discussed in earlier sections of this paper. Thus, the term $\mathbf{j} / \mu$ is an important quantity in plastic dynamics.

To clarify the physical meaning of $\mathbf{j} / \mu$, apply divergence to both-hand sides of Eq. (2). With the mathematical identity $\nabla \cdot(\nabla \times \boldsymbol{\omega})=0$ and the use of Eq. (4), this leads to

$$
\frac{\partial \rho}{\partial t}=-\frac{1}{\mu}(\nabla \cdot \mathbf{j})
$$

Equation (8) can be interpreted as follows. Substitution of Eq. (4) to Eq. (1) yields

$$
\rho=\varepsilon(\nabla \cdot \mathbf{v})=\nabla \cdot(\varepsilon \mathbf{v}) .
$$

The rightmost-hand side of Eq. (9) represents divergence of momentum density. Application of divergence theorem converts Eq. (8) to the integrated form as

$$
\frac{\partial}{\partial t} \iint \rho \mathrm{d} \Omega=-\iint \frac{\mathbf{j}}{\mu} \cdot \mathbf{n} \mathrm{d} S .
$$

The left-hand side of Eq. (10) represents change of the total momentum in a closed volume $\Omega$ over unit time and the right-hand side represents the cause of the change in momentum due to some action through the surface area. According to Newton's second law, change in momentum over time is the net external force. Thus, the right-hand side of Eq. (10) can be interpreted as the longitudinal external force acting on the closed volume via the surface of the volume; hence $\mathbf{j} / \mu$ is the external force on a unit volume. With this interpretation, consider Eq. (5) and notice that the external force $\mathbf{j} / \mu$ is the same direction as the acceleration $\partial \mathbf{v} / \partial t$. 
Equation (8) can also be viewed as an equation of continuity. From this viewpoint, $\mathbf{j} / \mu$ represents a flow of quantity $\rho=\varepsilon j^{0}=\varepsilon \nabla \cdot \mathbf{v}$. Being proportional to the symmetry charge $\nabla \cdot \mathbf{v}=j^{0}$ and formulaically equivalent to the electric charge density, $\rho$ is called the charge density. Equivalently speaking, $\mathbf{j} / \mu$ can be identified as the flow of charge across unit cross-sectional area. In the one-dimensional model of Figs. 9 and 10, this flow corresponds to motion of a stretched spring as a whole. In order for a stretched spring to move as a whole, the material at the tailing end must break as Fig. 9 illustrates, or becomes unstretchable (Fig. 10). The external force does not contribute further stretch of the spring, and therefore its work is not stored as spring energy; consequently, the energy is dissipated and therefore the deformation is not reversible. At the same time, the material on the other side of the broken spring viewed from the dynamic cross-head of the tensile machine loses its velocity (because it is no more pulled by a spring in the direction of tensile force). Consequently, the unit volume loses its total momentum. This is the physical mesomechanical picture of plastic strain.

\subsection{Physical mesomechanical interpretation}

Based on the fringe analysis and accompanying explanation with the spring model discussed above, it is possible to divide the development of plastic deformation under tensile loading at constant puling rate into the following stages.

Stage 1. Local spring breakage occurs and propagates

This is the initial stage of plastic deformation, which can occur while the stress-strain curve appears to be linear. At this stage, the spring breakage is limited to a local area. As the spring breaks, the stress relaxes in the region causing the neighboring region to be under high stress. This causes the local spring in the neighboring region to break, or the local spring breakage to propagate. If this stage of deformation is observed with a horizontally sensitive interferometer for a specimen being pulled horizontally, the fringes are tilted in the region where local spring breakage occurs. The fringe patterns observed in Experiment 1 indicates that in the parallel part of the specimen the initial spring breakage occurs at a side as Fig. 9 illustrates.

\section{Stage 2. Spring breakage propagates across specimen}

The propagation of local spring breakage stops when it reaches the other side of the specimen. Accordingly, the stress relaxation due to the spring breakage bridges across the width of the specimen. The fringes tilted by the abovementioned mechanism become vertical, and the total number of fringes exceeds the reference fringe number as discussed in Section 3.1.

\section{Stage 3. Specimen fractures at bridged location}

The initiation of plastic deformation in Stage 1 and the bridging effect in Stage 2 can occur at multiple locations of the same specimen. However, eventually, the cycle of this process ends. At that point, the final bridged location keeps stretching until the specimen fractures.

The repetition of the bridging process followed by fracture at the final bridged location is evidenced by a number of previous experiments [20,21,25] with the same type of interferometer as this study. In these experiments, the bridging effect is observed as a bright band pattern similar to the one in Fig. 3(e); the bright pattern shifts along the tensile axis till the specimen fractures at the location where the bright pattern gets stationary. In the case of this study, judging from the temporal change in the fringe patterns in Fig. 3, the bridging effect seems to occur at only one location near the horizontal center of the specimen where the bright spot appears once. In other experiment with the same specimen dimension and tensile speed, it has been observed that the bright pattern moves around the specimen if $2-5 \%$ of magnesium is mixed into aluminum. The condition regarding the mobility of this bright pattern is a subject of future study, but it seems that addition of other elements is a major factor.

From the viewpoints of physical mesomechanics and the stages of deformation argued above, the observations in Experiment 1 through 3 can be explained comprehensively. When a spring breakage and accompanying excess stretch occurs, the displacement field becomes rotational in such a way that the regions divided by the excess stretched location rotate in mutually opposite directions. In terms of the field Eq. (2), the situation is expressed by $\nabla \times \boldsymbol{\omega} \neq 0$ [26]. Under this condition, the restoring force $\nabla \times \omega / \mu$ is active as resisting mechanism against the tensile load, and the fringe patterns present this force in accordance with Eqs. (6) and (7). If the specimen is not plastically deformed previously, as is the case of Experiment 1, the fringe pattern presenting the $\nabla \times \boldsymbol{\omega} \neq 0$ feature of the displacement field is observed in Stage 1. In the region where the local spring breakage occurs, e.g., near the top edge of Fig. 3(b) where the fringe interval is more irregular than near the bottom edge, the $\nabla \times \omega / \mu$ force becomes the major component as the restoring mechanism; on the other hand, near the bottom edge of the specimen where $\nabla \times \omega$ is effectively zero, the longitudinal elastic force $\mathbf{j} / \mu$ is the dominant restoring mechanism. As the deformation enters Stage 2, the $\nabla \times \boldsymbol{\omega} \neq 0$ feature of the displacement field fades out, as the fringe pattern in Fig. 3(c) presents. At this point, across the entire width of the specimen, the longitudinal effect $\mathbf{j} / \mu$ enters the flow regime where it does not present the elastic force but instead represents the energy dissipation associated with the flow of $\rho$ defined by Eq. (9).

If the specimen is under a reload as Experiment 2 or 3, the situation depends both on the preload and reload stress level. Generally speaking, when the reload stress level is equal or lower than the preload stress level, the reload does not generate new permanent strain. Consequently, only the 
elastic portion of deformation is reproduced. According to the results of Experiment 2 and 3 under the condition of reload stress level equal to or lower than the preload stress, the corresponding fringe patterns dominantly represent rotational displacement. This is because of the rotational nature of the restoring force $\nabla \times \omega / \mu$.

The above observation can be clearly explained based on physical mesomechanics. From the physical mesomechanical viewpoint, the permanent strain is caused by the longitudinal effect being the flow of charge (Eq. (9)). While charges flow causing energy dissipation, the material still possesses elasticity represented by the restoring shear force $\nabla \times \omega / \mu$, and this shear force makes the displacement field rotational. When the preload stress level is in Stage 1, the material possesses the elasticity associated with shear force at various regions. Consequently, under reloading, the rotational nature of the displacement field is local, producing wavy fringe patterns like Fig. $6(a)$. When the preload stress level is higher, on the other hand, the material is unable to exert the restoring shear force in most part; as a result, the rotational feature of the displacement field under reloading becomes more global; in most cases, it is represented by a pair of opposite rotations as shown by Figs. $6(b, c)$.

Based on this argument and Eqs. (6) and (7), the horizontal component of shear force $F_{x}$ is found active near curved fringes such as the rightmost fringe in Fig. 3(b,c), and most part of Fig. $6(a)$. From the observation that the fringe slope $\partial \xi_{x} / \partial y$ varies along the $x$ axis, the vertical component $F_{y}$ appears to be active in Fig. 3(b) (except the rightmost curved fringe) and Fig. 6(a).

When the deformation enters Stage 3, the material is unable to exert the shear force at all, instead the current $\mathbf{j} / \mu$ flows. During this period, there is no stress increase; the stress-strain curve is flat. As Stage 1 and 2 resumes at another location of the specimen, in the case the bridging effect occurs at multiple locations, the stress start rising again and the same process described in the preceding paragraph repeats. When this repetition ends, or in the case where the material experiences Stage 1-3 process once like Experiment 1 of this study, the material loses the capability of exerting restoring force permanently as the deformation enters Stage 3. Once this happens, the material loses the mechanism to resist the external force and fractures.

\section{Conclusion}

In summary, this study demonstrates that at least for aluminum specimens under tensile loading, it is possible to reveal the loading hysteresis by reloading the specimen, monitoring the deformation with electronic speckle pattern interferometry, and examining the resultant interferometric fringe patterns. The fringe analysis with the help of physical mesomechanics indicates that development of deformation toward fracture can be divided into three stages. The fringe patterns formed by reloading the specimen at a stress level lower than the yield stress reveals (i) whether or not the specimen is previously loaded, and (ii) if previously loaded, what the stage of deformation under the previous load is. By increasing the reload stress level and observing the change in the overall fringe pattern, it is possible to estimate the stress level of the previous load quantitatively. Below, the characteristics feature of the fringe pattern for each stage is summarized. Here, the argument is for the case where the deformation is due to a horizontal tensile load and the electronic speckle pattern interferometry setup has sensitive to horizontal displacement. The same argument should be applicable to any combination of the orientation in the tensile axis and the interferometer's sensitivity.

In Stage 1, the irreversible atomic rearrangement occurs in a local zone, and propagates transversely to the tensile axis. This causes the opposite sides of the material bounded by the point of the local atomic rearrangement to rotate mutually opposite directions (called the segmental rotation). If the specimen experiences this process for the first time, this segmental rotation continues step-by-step as the irreversible atomic rearrangement propagates. When both the tensile axis and the interferometer sensitivity are horizontal, uniform elastic deformation generates equidistant, vertical parallel fringes. In this case, the step-by-step segmental rotation causes the fringes to tilt from the vertical position in the vicinity of the local atomic rearrangement. On the other hand, if a specimen is previously loaded to Stage 1, released from the load, and reloaded at a stress level equal or lower (than the previous load), the fringe pattern resulting from the reload presents a feature distinctively different from the step-by-step segmental rotation case; it consists of multiple fringe systems each representing accumulated segmental rotation. These fringe systems are characterized by equidistant, nearly horizontal parallel fringes. The overall fringe pattern appears somewhat wavy, indicating that it represents several local segmental bodily rotations.

In Stage 2, the irreversible atomic rearrangement completes its propagation across the width of the specimen. If the specimen is loaded for the first time, all fringes become basically vertical and the total number of fringes become greater than the reference fringe number, i.e., part of the specimen experiences contraction. If a specimen is previously loaded to Stage 2, released from the load, and reloaded at a stress level equal or lower, the resultant fringe system is drastically different from the previous load; it consists of multiple equidistant nearly horizontal patterns representing the accumulated segmental rotations as is the case when a specimen is previously loaded to Stage 1 . In other words, if a specimen is loaded for the first time to Stage 2, the overall fringe pattern is basically horizontal, and if the same specimen is reloaded after released from the previous load to Stage 2, the overall fringe pattern is basically horizontal. 
The overall fringe pattern of the nearly horizontal fringes observed by reloading a specimen previously deformed to Stage 2 is different from the case where the specimen is previously deformed to Stage 1. It normally consists of two fringe systems representing two segmental bodily rotations, each showing straighter fringes, as opposed to the wavy fringes in the case of previously deformed to Stage 1 . This indicates that when the previous deformation is in Stage 2, the plastic strain is more concentrated in a single point of the specimen, and therefore the material is barely deformed inside the segments.

If the same specimen loaded to Stage 2 is reloaded at a stress level higher than the previous load, the vertical fringes representing partial contraction resumes. In other words, when the reload stress level exceeds the previous load stress level, the specimen behaves as if it is loaded for the first time.

When the deformation enters Stage 3, the material completely loses the restoring mechanism. Consequently, the only mechanism to consume the external work done by the tensile machine is to dissipate the energy via flow of charges. When the flow ends, the material loses all mechanism to consume the external work, and fractures.

\section{Acknowledgment}

This study was supported in part by Yokohama National University's overseas faculty training program.

\section{References}

[1] P.K. Rastogi, Speckle Metrology, Ed. by R.S. Sirohi, Marcel Dekker, New York, 1993.

[2] A. Kato, Y.Z. Dai, and F.P. Chiang, Damage monitoring of metal materials by laser speckle assisted by image processing techniques, JSME Int. I, 34 (1991) 374.

[3] A.J. Moore and J.R. Tyrer, The evaluation of fracture mechanics parameters from ESPI fringe patterns, J. Strain Analysis, 29 (1994) 257.

[4] S. Toyooka, Dynamic observation of metal plastic deformation process using ESPI method, Met. Technol., 70 (2000) 869.

[5] A. Kato, Detection of fatigue damage in steel using laser speckle, Opt. Laser. Eng., 33, (2000) 323.

[6] R. Wang and M. Kido, High temperature fatigue deformation behaviors of thermally sprayed steel measured with electronic speckle pattern interferometry method, Mater. Research Bulletin, 38 (2003) 1401.

[7] F.V. Diaz and A.F. Armas, and G.H. Kaufmann, Nondestructive evaluation of the fatigue damage accumulation process around a notch using a digital image measurement system, Opt. Laser. Eng., 41 (2004) 477.
[8] Y. Shinhara, R. Wang, T. Tokuda, M. Kido, and Y. Harada, Surface Strain Measurement of Thermally Sprayed Ceramics Coatings under Static and Cyclic Loadings and Its Application to Detection of Delamination, Mater. Trans., 48 (2007) 769.

[9] E.E. Sackett, L. Germain, and M.R. Bache, Crystal plasticity, fatigue crack initiation and fatigue performance of advanced titanium alloys, Int. J. Fatigue, 29 (2007) 2015.

[10] F.P. Chiang, Super-resolution digital speckle photography for micro/nano measurements, Opt. Laser. Eng., 47 (2009) 274.

[11] A. Davila, G. Garnica, J.A. Lopez, and F.J. Carrion, Fatigue damage detection using a speckle-contrast technique, Opt. Laser. Eng., 47 (2009) 398.

[12] T. Mita, Observation of Crack Tip Vicinity Using Electronic Speckle Pattern Interferometry: Master's Thesis 04GMM 23, Tokyo Denki University, 2004.

[13] Physical Mesomechanics of Heterogeneous Media and ComputerAided Design of Materials, Ed. by V.E. Panin, Cambridge International Science, Cambridge, 1998.

[14] V.E. Egorushkin, Dynamics of plastic deformation: Waves of localized plastic deformation in solids, Russ. Phys. J., 35, No. 4 (1992) 316.

[15] V.E. Panin, Yu.V. Grinyaev, V.E. Egorushkin, I.L. Bukhbinder, and S.N. Kul'kov, Excited state spectrum and vortex mechanical field in a deformable crystal, Russ. Phys. J., 30, No. 1 (1987) 24.

[16] S. Yoshida, T. Sasaki, and G.A. Gaffney, Dynamics of Plastic Deformation Based on a Field Theory, in Proc. SEM X Int. Congress, June 1-4 2009, Albuquerque, NM USA.

[17] S. Yoshida, G.A. Gaffney, and K. Yoshida, Revealing load hysteresis based on physical-mesomechanical deformation and fracture criteria, Phys. Mesomech., 13, No. 5-6 (2010) 337.

[18] J.A. Leendertz, Interferometric displacement measurement on scattered surface utilizing speckle effect, J. Phys. E, 3 (1970) 214.

[19] S. Yoshida, Suprapedi, R. Widiastuti, E.T. Astuti, and A. Kusnowo, Phase evaluation for electronic speckle-pattern interferometry deformation analyses, Opt. Lett., 20 (1995) 755.

[20] S. Yoshida, M.I. Muhamad, R. Widiastuti, and A. Kusnowo, Optical interferometric technique for deformation analysis, Opt. Exp., 2 (1998) 516 .

[21] S. Yoshida, M.I. Muhamed, M. Pardede, R. Widiastuti, M.B. Siahaan, and A. Kusnowo, Optical interferometry applied to analyze deformation and fracture of aluminum alloys, Theor. Appl. Fract. Mech., 27 (1997) 85.

[22] I.J.R. Aitchson and A.J.G. Hay, Gauge Theories in Particle Physics, IOP Publishing, Bristol, 1989.

[23] S. Yoshida, Field theoretical approach to dynamics of plastic deformation and fracture, AIP Conf. Proc., 1186 (2009) 108.

[24] S. Yoshida, Dynamics of plastic deformation based on restoring and energy dissipative mechanisms in plasticity, Phys. Mesomech., 11, No. 3-4 (2008) 137.

[25] S. Yoshida, B. Siahaan, M.H. Pardede, N. Sijabat, H. Simangunsong, T. Simbolon, and A. Kusnowo, Observation of plastic deformation wave in a tensile-loaded aluminum-alloy, Phys. Lett. A, 251 (1999) 54.

[26] S. Yoshida, Consideration on fracture of solid-state materials, Phys. Lett. A, 270 (2000) 320. 